\title{
A SPECTRAL SEQUENCE FOR THE INTERSECTION OF SUBSPACE PAIRS
}

\author{
RICHARD N. CAIN
}

\begin{abstract}
A general-homology spectral sequence that generalizes the Mayer-Vietoris exact sequence is established between the intersection of a family of subspace pairs and the system of partial unions of the family. The basis of the construction is a topological analogue of the "bar construction" of homological algebra.
\end{abstract}

We shall show here that a finite family $\mathscr{P}=\left\{\left(X_{i}, A_{i}\right) \mid i \in I\right\}$ of subspace pairs ${ }^{1}$ in a space $X$ have, for each general homology theory $h_{*}$, a spectral sequence

$$
E_{1 ; j}^{n} \cong \bigoplus_{N s=n} h_{j}\left(\bigcup_{s} X_{i}, \bigcup_{s} A_{i}\right) \overrightarrow{\vec{n}} h_{j-n}\left(\bigcap_{I} X_{i}, \bigcap_{I} A_{i}\right)
$$

$(s \subset I), N s$ being (number of members in $s)-1$. This is just the spectral sequence of a cover with the roles of union and intersection interchanged. Its connection with the Mayer-Vietoris sequence will be examined below, and we shall derive from it the spectral sequence of the homology sheaf of $X$.

Construction of (a). Start with any finite set $U$ ( $=$ the universe) that contains $I$ as a subset, and define (using $T$ to denote the based unit interval, while $\bigwedge_{J} Y=Y^{J} /\left\{\eta \in Y^{J} \mid * \in \eta J\right\}$ for based spaces $Y$ and finite sets $J$ )

(b)

$$
\begin{aligned}
\nabla s & =\bigwedge_{s} \partial T \wedge \bigwedge_{U-s} T \quad(s \subset U), \\
K & =\bigcup_{a \in U} \nabla\{a\}=\partial \nabla \varnothing, \\
C & =\{*\} \cup \bigcup_{a \in U-I} \nabla\{a\}=\bigwedge_{I} T \wedge \partial \bigwedge_{U-I} T, \\
M & =X \times C \cup \bigcup_{s \subset I} \bigcup_{s} A_{i} \times \nabla s, \\
L^{n} & =M \cup \bigcup_{s \subset I ; N s \geq n} \bigcup_{s} X_{i} \times \nabla s \quad(n \in Z) \\
L & =L^{0}=X \times C \bigcup_{s \in I} \bigcup_{s} X_{i} \times \nabla s .
\end{aligned}
$$

Received by the editors February 16, 1973.

AMS (MOS) subject classifications (1970). Primary 55H05, 55H25; Secondary 55C05.

Key words and phrases. Spectral sequence, general homology, general cohomology, cover, duality.

${ }^{1}$ Assumed to be subcomplex pairs under some CW complex structure on $X$.

(c) American Mathematical Society 1974 
We require $I \neq \varnothing$ but permit $U=I$. Caution. Here $\partial$ is used in the context of based spaces, so $\partial \partial T=\{*\}$, not $\varnothing$. The formulas for the spectral sequence are as follows (as in [1, p. $108 \mathrm{ff}$.] for any filtered space):

$$
\begin{array}{r}
E_{r ; j}^{n}=\frac{\operatorname{Im}\left[h_{p}\left(L^{n}, L^{n+r}\right) \rightarrow h_{p}\left(L^{n-r+1}, L^{n+r}\right)\right]}{\operatorname{Im}\left[h_{p}\left(L^{n+1}, L^{n+r}\right) \rightarrow h_{p}\left(L^{n-r+1}, L^{n+r}\right)\right]} \quad(n, j \in Z ; r=1,2, \cdots) \\
\quad \text { where } p=j-n+N U \text { and maps are induced by inclusion, }
\end{array}
$$

(c) $d_{r ; j}^{n}=$ homomorphism $E_{r ; j}^{n} \rightarrow E_{r ; j+r-1}^{n+r}$ induced by $\partial_{p}$ for the triple $\left(L^{n}, L^{n+r}, L^{n+2 r}\right)$,

$u_{r ; j}^{n}=$ isomorphism $E_{r+1 ; j}^{n} \rightarrow \mathscr{H}_{j}^{n} E_{r ; *}^{*}$ induced by $h_{p}(\subset)$ for the inclusion $\left(L^{n}, L^{n+r+1}\right) \subset\left(L^{n}, L^{n+r}\right)$.

Thus, $E_{\infty ; j}^{n}=E_{r ; j}^{n}$ for large $r,=F^{n} G_{j-n} / F^{n+1} G_{j-n}$, where

(c, Cont'd.)

$$
G_{q}=h_{a+N U}(L, M) \quad(q \in Z),
$$

$$
F^{n} G_{q}=\operatorname{Im}\left[h_{a+N U}\left(L^{n}, M\right) \rightarrow h_{a+N U}(L, M)\right] .
$$

(Note that $G_{*}=F^{0} G_{*} \supset F^{1} G_{*} \supset \cdots \supset F^{N I+1} G_{*}=\{0\}$.)

Define also, for each $a \in U$ and $s \subset U$ containing $a$,

$$
\begin{gathered}
v(a): \boldsymbol{h}_{q}(\nabla s, \partial \nabla s) \underset{\text { excision }}{\cong} \boldsymbol{h}_{q}\left(\partial \nabla s^{\prime}, \partial \nabla s^{\prime}-\nabla^{\#} s\right)\left(s^{\prime}=s-\{a\}\right), \\
\mu(a): \boldsymbol{h}_{a+1}\left(\nabla s^{\prime}, \partial \nabla s^{\prime}\right) \stackrel{\partial_{q+1}}{\cong} h_{a}\left(\partial \nabla s^{\prime}, \partial \nabla s^{\prime}-\nabla^{\#} s\right), \\
\sigma(a)=\mu(a)^{-1} v(a),
\end{gathered}
$$

where $\nabla^{\#}(\cdot)=\nabla(\cdot)-\partial \nabla(\cdot)$ and $\boldsymbol{h}_{*}=$ any general homology theory, $q$ any integer. For distinct $a_{1}, \cdots, a_{k} \in s \subset U(k \geqq 1)$, denote $s-\left\{a_{1}, \cdots, a_{k}\right\}$ as $s^{\prime \prime}$ and define

$$
\text { (d, Cont'd.) } \quad \sigma\left(a_{*}\right): \boldsymbol{h}_{q}(\nabla s, \partial \nabla s) \stackrel{\cong}{\longrightarrow} \boldsymbol{h}_{q+k}\left(\nabla s^{\prime \prime}, \partial \nabla s^{\prime \prime}\right)
$$

as $\sigma\left(a_{k}\right) \cdots \sigma\left(a_{2}\right) \sigma\left(a_{1}\right)$, where $a_{*}$ means $\left(a_{1}, \cdots, a_{n}\right) . \sigma\left(a_{*}\right)$ is alternating, because, for any permutation of $a_{*}$, the corresponding coordinate transformation of $\nabla s^{\prime \prime}$ permutes the factors of $\sigma\left(a_{*}\right)$ in the same way.

Now let $a_{*}=\left(a_{0}, \cdots, a_{N U}\right)$ be a choice of numbering of $U$, and for each nonempty subset $s \subset I$ let $i_{*}^{s}=\left(i_{0}^{s}, \cdots, i_{N s}^{s}\right)$ be a choice of numbering of $s$. They, together with $\sigma$ and Lemmas 1, 2 below, determine two 
isomorphisms:

(e)

$$
\begin{aligned}
& \begin{array}{r}
\left.\cong_{I} X_{i}, \bigcap_{I} A_{i}\right) \\
\cong \downarrow^{(-1)^{j N N \sigma} \sigma\left(a_{*}\right)}
\end{array} \\
& r h_{a+N U+1}\left(\left(\bigcap_{I} X_{i}, \bigcap_{I} A_{i}\right) \times(\nabla \varnothing, \partial \nabla \varnothing)\right) \\
& \text { (Lemma 1) } \cong \downarrow^{\partial_{a+N V+1} \text { of the homology theory }} \operatorname{h}_{*}=h_{*}\left(\left(\cap_{I} X_{i}, \cap_{I} A_{i}\right) \times(\cdot)\right)
\end{aligned}
$$

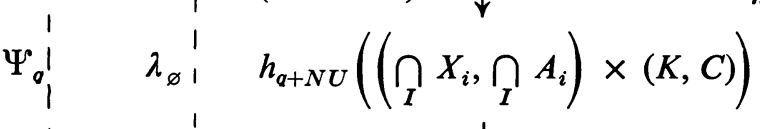

$$
\begin{aligned}
& \text { (Lemma 1) } \cong h^{h_{a+N U}(C)} \\
& h_{a+N U}(L, M)
\end{aligned}
$$

$$
\begin{aligned}
& i^{---------\bigoplus_{N s=n ; s \subset I}} h_{j}\left(\bigcup_{s} X_{i}, \bigcup_{s} A_{i}\right) \\
& \cong \downarrow(-1)^{j N U \cdot\left(\oplus \sigma\left(i_{*}^{8}\right)-1 \sigma\left(a_{*}\right)\right)} \\
& r_{i s=n ; s \subset I}^{---\bigoplus_{p}} h_{p}\left(\left(\bigcup_{s} X_{i}, \bigcup_{s} A_{i}\right) \times(\nabla s, \partial \nabla s)\right) \\
& \text { (Lemma 2) } \cong \downarrow h_{p}(\subset)
\end{aligned}
$$

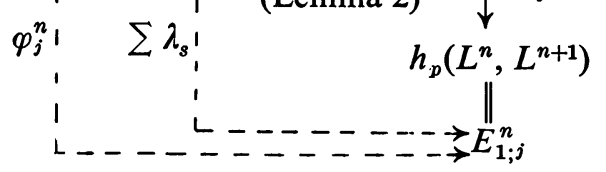

$(q, n, j \in Z)$ which combine with formulas (c) to give the formula (a).

LEMMA 1. $\lambda_{\varnothing}$ is an isomorphism.

Proof. (Referring to (e).) $\partial_{q+N U+1}$ is bijective by contractibility of $(\nabla \varnothing, C)=\bigwedge_{I} T \wedge\left(\bigwedge_{U-I} T, \partial \bigwedge_{U-I} T\right)$. For bijectivity of $h_{a+N U}(\subset)$ it suffices by the Five Lemma to consider the case $A_{i}=\varnothing$ (all $i \in I$ ). Define

$$
\begin{gathered}
L^{(n)}=X \times C \cup \underset{s \subset I ; N s \geqq n}{\cup}\left[\bigcap_{s} X_{i} \times \bigcup_{s} \nabla\{i\}\right] \quad(n \in Z) . \\
h_{*}\left(L^{(n)}, L^{(n+1)}\right) \cong \bigoplus_{N s=n} h_{*}\left(\left(\bigcap_{s} X_{i}, \bigcup_{I-s} X_{i} \cap \bigcap_{s} X_{i}\right) \times\left(C \cup \bigcup_{s} \nabla\{i\}, C\right)\right)
\end{gathered}
$$


by additivity of homology, $\cong\{0\}$ by contractibility of

$$
\left(C \cup \bigcup_{s} \nabla\{i\}, C\right)=\bigwedge_{I-s} T \wedge\left(\partial \underset{s \cup(U-I)}{\bigwedge_{s}} T, \bigwedge_{s} T \wedge \partial \bigwedge_{U-I} T\right),
$$

assuming $0 \leqq n<N I . h_{*}\left(\bigcap_{I} X_{i} \times(K, C)\right) \cong h_{*}\left(L^{(N I)}, X \times C\right)$ by excision, $\cong h_{*}\left(L^{(N I-1)}, X \times C\right) \cong \cdots \cong h_{*}\left(L^{(0)}, X \times C\right)=h_{*}(L, X \times C)$ by exactness using above $\{0\}$.

LEMMA 2. $\sum \lambda_{s}$ is an isomorphism.

Proof. Additivity of homology.

Comparison with the Mayer-Vietoris sequence. Since (a) relates the various unions of the pairs $\mathscr{P}$ to their intersection it brings to mind the Mayer-Vietoris sequence. (a) is in fact a generalization of the latter, as we shall now show. (The Mayer-Vietoris sequence is the $N I=1$ case of $\varepsilon, \delta^{0}, \beta$ below.)

For any $n \in Z$ let $S_{n}(I)=\left\{i_{*}=\left(i_{0}, \cdots, i_{n}\right) \mid i_{0}, \cdots, i_{n} \in I\right\}$, which is to entail that $S_{n}(I)=\varnothing$ for negative $n$. Using $X_{i_{*}}$ to mean $X_{i_{0}} \cup \cdots \cup X_{i_{n}}$, define $C^{n}\left(\mathscr{P} ; h_{j}(\bigcup \cdot)\right)(j \in Z)=$ subgroup of $\prod_{i_{*} \in S_{n}(I)} h_{j}\left(X_{i_{*}}, A_{i_{*}}\right)$ consisting of alternating members $\xi=\left\{\xi^{i *} \mid i_{*} \in S_{n}(I)\right\}$ for which $\xi^{i_{*}}=0$ whenever two or more of $i_{0}, \cdots, i_{n}$ are equal, and note that

$$
C^{n}\left(\mathscr{P} ; h_{j}(\bigcup \cdot)\right) \cong \underset{N s=n ; s \subset I}{\bigoplus_{s}} h_{j}\left(\bigcup_{s} X_{i}, \bigcup_{s} A_{i}\right)
$$

under the correspondence $\xi \mapsto\left\{\xi^{i_{*}} \mid N s=n, s \subset I\right\}$. Denote by $\Phi_{j}^{n}$ the composite of $\varphi_{j}^{n}$ with this isomorphism.

LEMMA 3. The following diagram commutes:

$$
\begin{aligned}
& h_{j}\left(\bigcap_{I} X_{i}, \bigcap_{I} A_{i}\right) \stackrel{\varepsilon}{\longrightarrow} C^{0}\left(\mathscr{P} ; h_{j}(\bigcup \cdot)\right) \stackrel{\delta^{0}}{\longrightarrow} C^{1}\left(\mathscr{P} ; h_{j}(\bigcup \cdot)\right) \stackrel{\delta^{1}}{\longrightarrow} \cdots \\
& \cong \downarrow^{\Psi_{j}} \quad \quad \cong \downarrow^{\kappa_{j}} \quad \cong \Phi^{0} \quad \ldots
\end{aligned}
$$

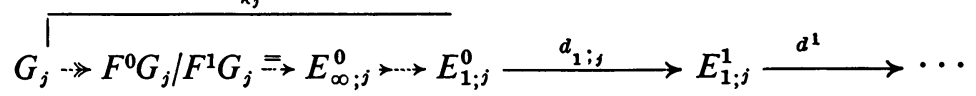

where

and

$$
\varepsilon(\xi)^{i_{0}}=\left.\xi\right|^{X_{i_{0}}, A_{i_{0}}} \quad\left(\xi \in h_{j}\left(\bigcap_{I} X_{i}, \bigcap_{I} A_{i}\right), i_{0} \in I\right)
$$

$$
\begin{aligned}
\delta^{n}(\xi)^{i_{*}}=\left.\sum_{0 \leqq k \leqq n+1}(-1)^{k} \xi^{i_{*}(k)}\right|^{X_{i_{*}}, A_{i_{*}}} & \\
& \left(n \in Z, \xi \in C^{n}\left(\mathscr{P} ; h_{j}(\bigcup \cdot)\right), i_{*} \in S_{n+1}(I)\right)
\end{aligned}
$$

$i_{*}(k)$ being $\left(i_{0}, \cdots, i_{k-1}, i_{k+1}, \cdots, i_{n+1}\right)$. 
Proof. For the square involving $\delta^{n}$ for some $n, 0 \leqq n \leqq N I$, we consider an arbitrary element of $C^{n}\left(\mathscr{P} ; h_{j}(\bigcup \cdot)\right)$ of the form $\chi\left(i_{*} ; \theta\right)$ defined as follows: $i_{*}$ is a numbering of a subset $s \subset I$ with $N s=n, \theta$ belongs to $h_{j}\left(\bigcup_{s} X_{i}, \bigcup_{s} A_{i}\right)$, and $\chi\left(i_{*} ; \theta\right)^{i_{*}^{\prime}}= \pm \theta$ or 0 , depending upon whether $i_{*}^{\prime}$ is an even or odd permutation of $i_{*}$ or not a permutation of $i_{*}$, respec-

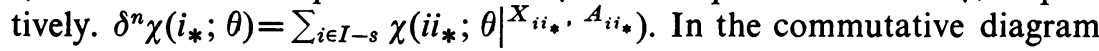

$$
\boldsymbol{h}_{p}(\nabla s, \partial \nabla s) \stackrel{\{\mu(i)\}}{\longrightarrow} \oplus \boldsymbol{h}_{p-1}\left(\partial \nabla s, \partial \nabla s-\nabla^{\#}(s \cup i)\right) \stackrel{\oplus v(i)}{\cong}
$$

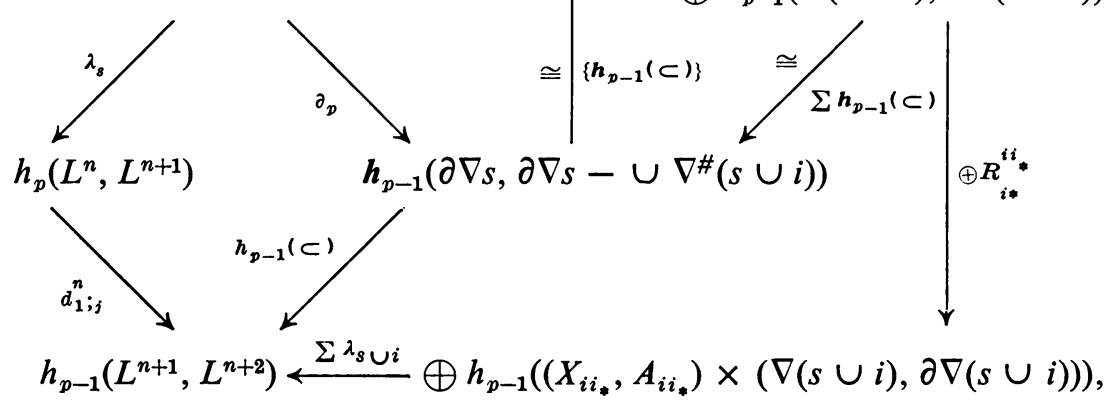

assume $h_{*}=h_{*}\left(\left(X_{i_{*}}, A_{i_{*}}\right) \times(\cdot)\right)$, that each $R_{i_{*}}^{i i_{*}}$ is induced by the appropriate inclusion, and each sum or union is taken over $\{i \mid i \in I-s\}$. We have

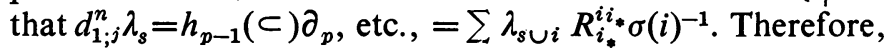

$$
\begin{aligned}
(-1)^{j N U} d_{1 ; j}^{n} \Phi_{j}^{n} \chi\left(i_{*} ; \theta\right) & =d_{1 ; j}^{n} \lambda_{s} \sigma\left(i_{*}\right)^{-1} \sigma\left(a_{*}\right) \theta \\
& =\sum \lambda_{s \cup i} R_{i_{*}}^{i i_{*}} \sigma\left(i i_{*}\right)^{-1} \sigma\left(a_{*}\right) \theta \\
& =\sum \lambda_{s \cup i} \sigma\left(i i_{*}\right)^{-1} \sigma\left(a_{*}\right)\left(\left.\theta\right|^{\left.X_{i i_{*}}, A_{i i_{*}}\right)}\right. \\
& =(-1)^{j N U} \cdot \Phi_{j}^{n+1} \delta^{n} \chi\left(i_{*} ; \theta\right) .
\end{aligned}
$$

We have thus proved the square commutative, since the $\chi\left(i_{*} ; \theta\right)$ 's generate $C^{n}\left(\mathscr{P} ; h_{j}(\bigcup \cdot)\right)$. For the square involving $\varepsilon$, the same argument works with $h_{*}$ redefined as $h_{*}\left(\left(\bigcap_{I} X_{i}, \bigcap_{I} A_{i}\right) \times(\cdot)\right), s$ replaced by $\varnothing$, and $d_{1 ; j}^{n}$ replaced by $\kappa_{q}$.

Lemma 4. If $A_{i}=\varnothing$ (all $\left.i \in I\right)$, the following diagram commutes:

$$
\begin{aligned}
& C^{N I}\left(\mathscr{P} ; h_{j}(\bigcup \cdot)\right) \longrightarrow h_{j-N I}\left(\bigcap_{I} X_{i}\right) \\
& \cong \downarrow_{j}^{N I} \quad \cong \downarrow^{N-N I} \\
& E_{1 ; j}^{N I} \rightarrow E_{\infty ; j}^{N I} \rightrightarrows F^{N I} G_{j-N I} / F^{N I+1} G_{j-N I} \nrightarrow G_{j-N I}
\end{aligned}
$$


where $\beta$ has the formula

$$
\begin{aligned}
& C^{N I}\left(\mathscr{P} ; h_{j}(\bigcup \cdot)\right) \cong h_{j}\left(\bigcup_{I} X_{i}\right) \rightarrow h_{j}\left(\bigcup_{I} X_{i}, \bigcup_{j<N I} X_{(j)}\right) \\
& \underset{\text { excision }}{\stackrel{\cong}{\longrightarrow}} h_{j}\left(X_{(N I)}, \bigcup_{j<N I} X_{(j)} \cap X_{(N I)}\right)
\end{aligned}
$$

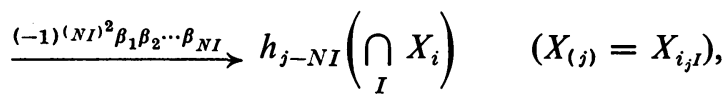

each $\beta_{k}(1 \leqq k \leqq N I)$ being the composite

$$
\begin{gathered}
h_{j-N I+k}\left(\bigcap_{j \geqq k} X_{(j)}, \bigcup_{j<k} X_{(j)} \cap \bigcap_{j \geqq k} X_{(j)}\right) \\
\downarrow^{\partial_{j-N I+k}} \\
h_{j-N I+k-1}\left(\bigcup_{j<k} X_{(j)} \cap \bigcap_{j \geqq k} X_{(j)}, \bigcup_{j<k-1} X_{(j)} \cap \bigcap_{j \geqq k} X_{(j)}\right) \\
\cong \downarrow \downarrow \text { excision } \\
h_{j-N I+k-1}\left(\bigcup_{j \geqq k-1} X_{(j)}, \bigcup_{j<k-1} X_{(j)} \cap \bigcap_{j \geqq k-1} X_{(j)}\right) .
\end{gathered}
$$

Proof. Omitted. Consists of comparing each $\beta_{k}$ with the appropriate form of $\sigma\left(i_{j}^{I}\right)^{-1}$ in one large commutative diagram.

Independence from $U$. Let $U^{+}=U \oplus\{a\}$ for some point $a$ apart from $U$, and indicate by a superscript + the $U^{+}$-version of each of the notions (b)-(e). To prove that the choice of $U$ is immaterial it suffices to prove $(\mathrm{c}) \cong\left(\mathrm{c}^{+}\right),(\mathrm{e}) \cong\left(\mathrm{e}^{+}\right)$. We therefore define an isomorphism

$$
l^{n, m}: h_{p}\left(L^{n}, L^{m}\right) \rightarrow h_{p^{+}}\left(L^{+n}, L^{+m}\right)
$$

as follows, for $n, j$ as in (c) and $m \geqq n$ :

$$
\begin{aligned}
h_{p}\left(L^{n}, L^{m}\right)=h_{p}^{(n, m)} & (\partial \underset{\{a\}}{\bigwedge} T,\{*\}) \\
& \stackrel{\partial_{p+1}^{-1}}{\cong} h_{p+1}^{(n, m)}\left(\bigwedge_{\{a\}} T, \partial \bigwedge_{\{a\}} T\right) \underset{\text { excision }}{\cong} h_{p^{+}}\left(L^{+n}, L^{+m}\right) .
\end{aligned}
$$

Here $\boldsymbol{h}_{*}^{(n, m)}$ is the general homology theory of based compact pairs $(Y, B)$ with formula $h_{*}^{(n, m)}(Y, B)=h_{*}\left(L^{n}(Y), L^{m}(Y) \cup L^{n}(B)\right), \quad L^{n}(Y)$ being $X \times C \wedge Y \cup \bigcup_{s \subset I}\left[\bigcup_{s} A_{i} \times \nabla s \wedge Y\right] \cup \bigcup_{s \subset I ; N s \geqq n}\left[\bigcup_{s} X_{i} \times \nabla s \wedge Y\right]$. Then, $(-1)^{j-n-1} l^{n, n+r}$ induces an isomorphism $E_{r ; j}^{n} \rightarrow E_{r ; j}^{+n}(n, j \in Z ; r=1$, $2, \cdots)$ that carries $d_{r ; j}^{n}$ into $d_{r ; j}^{+n}, \Phi_{j}^{n}($ for $r=1)$ into $\Phi_{j}^{+n}$, etc., as required. We assume that $a_{*}^{+}=a_{*} a$. 
Functoriality. Constructing (a) is more difficult than constructing the spectral sequence of a cover in that the underlying spaces (b) do not depend functorially on $(X, I, \mathscr{P})$. $U$ has been introduced as a remedy.

We assume that a morphism from $(X, I, \mathscr{P})$ to another such triple $(Y, J, \mathscr{Q}), \mathscr{Q}$ being a finite family $\left\{\left(Y_{j}, B_{j}\right) \mid j \in J\right\}$ of subspace pairs in a space $Y$, is a map $f: X \rightarrow Y$ of spaces together with a map $\pi: J \rightarrow I$ of sets such that $\left(f X_{\pi j}, f A_{\pi j}\right) \subset\left(Y_{j}, B_{j}\right) \quad(j \in J)$. Evidently $C^{n}\left(\mathscr{P} ; h_{q}(\bigcup \cdot)\right)$ $(n, q \in Z)$ depends functorially on $(X, I, \mathscr{P})$ if $(f ; \pi)$ is regarded as inducing the map $C^{n}(f ; \pi): C^{n}\left(\mathscr{P} ; h_{q}(\bigcup \cdot)\right) \rightarrow C^{n}\left(\mathscr{Q} ; h_{q}(\bigcup \cdot)\right)$ with the formula $\left(C^{n}(f ; \pi) \xi\right)^{j_{*}}=h_{q}(f ; \pi)^{j_{*}} \xi^{\pi j} * \quad\left(\xi \in C^{n}\left(\mathscr{P} ; h_{q}(\bigcup \cdot)\right), j_{*} \in S_{n}(J)\right)$, $h_{q}(f ; \pi)^{j_{*}}$ being the homomorphism $h_{q}\left(X_{\pi j_{*}}, A_{\pi j_{*}}\right) \rightarrow h_{q}\left(Y_{j_{*}}, B_{j_{*}}\right)$ induced by $\left.f\right|_{X_{\pi j}}$. Similarly, $h_{*}\left(\bigcap_{I} X_{i}, \bigcap_{I} A_{i}\right)$ is functorial, the induced map to be denoted $h_{*}(f ; \bigcap)$.

Let primes signify the $(Y, J, \mathscr{Q})$-version of the notions (b)-(e). To show that $(\mathrm{c}), \Psi_{*}, \Phi_{*}^{*}$ depend functorially on $(X, I, \mathscr{P})$, we need only produce a homomorphism of (c) to $\left(c^{\prime}\right)$ which, when considered along with $C^{*}(f ; \pi)$ and $h_{*}(f ; \bigcap)$, maps $\Psi_{*}, \Phi_{*}^{*}$ to $\Psi_{*}^{\prime}, \Phi_{*}^{\prime *}$ respectively. It is easy to see that this map of (c) to ( $\left.\mathrm{c}^{\prime}\right)$ is a fortiori unique and functorially dependent on the morphism $(f ; \pi)$.

We start by assuming $U^{\prime}=U \supset I \oplus J$. Define $\omega: \bigwedge_{U} T \rightarrow \bigwedge_{U} T$ to be the involution $\bigwedge_{U-(\pi J \cup J)} 1_{T} \wedge \bigwedge_{\pi J} \omega_{i}$, where, for each $i \in \pi J$,

$$
\omega_{i}\left(t_{i} \wedge \bigwedge_{\pi^{-1}\{i\}} t_{j}\right)=m \wedge \bigwedge_{\pi^{-1}\{i\}}\left(t_{j} t_{i} / m\right)
$$

$\left(t_{i}, t_{j} \in T\right.$ for $\left.j \in \pi^{-1}\{i\}\right), m$ being $\operatorname{Max}_{\pi^{-1}\{i\}} t_{j}$. It is easily shown that $\omega \nabla\{i\}=\bigcup_{\pi^{-1}\{i\}} \nabla\{j\}$ for $i \in \pi J$, while $\omega \nabla\{i\} \subset C^{\prime}$ for $i \in I-\pi J$. The consequence is $(f \times \omega) L^{n} \subset L^{\prime n}(n \in Z)$, with an induced homomorphism $l^{n, m}: h_{p}\left(L^{n}, L^{m}\right) \rightarrow h_{p}\left(L^{\prime n}, L^{\prime m}\right)(m \geqq n)$. The map $(-1)^{\text {number of members in } \pi_{J}}$. $l^{n, n+r}$ induces the required $E_{r ; j}^{n} \rightarrow E_{r ; j}^{\prime n}(n, j \in Z ; r=1,2, \cdots)$. (The power of $(-1)$ is the degree of $\omega$.

The homology sheaf. Let $\mathscr{P}=\left\{\left(X, A \cup\left(X-U^{i}\right)\right) \mid i \in I\right\}=\mathscr{P}_{\mathscr{U}}$ for some finite open cover $\mathscr{U}=\left\{U^{i} \mid i \in I\right\}$ of $X, A$ being some subspace. Evidently

$$
\begin{aligned}
& h_{*}\left(\bigcap_{I} X_{i}, \bigcap_{I} A_{i}\right)=h_{*}(X, A), \\
& C^{*}\left(\mathscr{P}_{\mathscr{U}} ; h_{*}(\bigcup \cdot)\right)=C^{*}\left(\mathscr{U} ; h_{*}^{X, A}\right),
\end{aligned}
$$

where $h_{*}^{X, A}$ is the graded presheaf $\left\{h_{*}(X, A \cup(X-\mathcal{O})) \mid\right.$ open $\left.\mathcal{O} \subset X\right\}$. Thus, we obtain a spectral sequence

$$
E_{2 ; j}^{n} \cong H^{n}\left(\mathscr{U} ; h_{j}^{X, A}\right) \overrightarrow{\vec{n}} h_{j-n}(X, A) .
$$


For $X$ compact, the direct limit of (f), as $\mathscr{U}$ is refined, is a spectral sequence

$$
E_{2 ; j}^{n} \cong H^{n}\left(X ; h_{j}^{X, A}\right) \underset{n}{\Rightarrow} h_{j-n}(X, A),
$$

where $h_{j}^{X, A}$ is the induced sheaf of $h_{j}^{X, A}$. As $A$ approximates an open set $V$ from within, the direct limit of $(\mathrm{g})$ is

$$
E_{2 ; j}^{n} \cong H^{n}\left(X, V ; h_{j}^{X}\right) \underset{\vec{n}}{\Rightarrow} h_{j-n}(X, V) \text {. }
$$

$h_{*}^{X}$ is called the homology sheaf of $X$. If $h_{j}^{X} \cong\{0\}$ except for $j=j_{0}$ (= some integer), e.g., if $X$ is a $j_{0}$-manifold and $h_{*}$ is standard, then (h) collapses to a family of isomorphisms

$$
H^{n}\left(X, V ; h_{j_{0}}^{X}\right) \cong h_{j_{0}-n}(X, V) \quad(n \in Z)
$$

(Compare to [2].)

\section{REFERENCES}

1. J. T. Schwartz, Differential geometry and topology, Courant Inst. of Math. Sci. Report, New York, 1966, Gordon and Breach, New York, 1968.

2. E. C. Zeeman, Dihomology. III. A generalization of the Poincaré duality for manifolds, Proc. London Math. Soc. (3) 13 (1963), 155-183. MR 27 \#2980c.

Department of Mathematics, Carnegie-Mellon University, Pittsburgh, Pennsylvania 15213

Current address: 411 South Graham Street, Pittsburgh, Pennsylvania 15232 